\title{
REAL ALGEBRAIC VARIETY STRUCTURES ON P. L. MANIFOLDS
}

\author{
BY SELMAN AKBULUT AND HENRY C. KING ${ }^{1}$
}

Communicated by P. T. Church, October 25, 1976

A closed smooth manifold $M^{m}$ is said to bound a smooth spine-manifold if $M$ bounds a compact smooth manifold $W^{m+1}$ and if there are a finite number of transversally intersecting closed submanifolds $\left\{M_{i}\right\}$ of $W$ such that $W / U M_{i}$ $\approx$ cone $(M)$, where $\approx$ is piecewise differentiable homeomorphism.

DEFinition. An $A_{1}$-structure on a P. L. manifold $M^{m}$ is: $M=M_{0} \cup$ $\bigcup_{i}$ cone $\left(\Sigma_{i}\right) \times N_{i}$ where $M_{0}$ is a codimension zero smooth submanifold of $M$, $\partial M_{0}=\mathrm{II}_{i} \Sigma_{i} \times N_{i}, N_{i}$ 's are smooth manifolds and $\Sigma_{i}$ 's are exotic spheres bounding smooth spine-manifolds.

$A_{1}$-structures satisfy regular neighborhood and product structure properties, and there is a classifying space $B_{A_{1}}$ with inclusions $B_{0} \rightarrow B_{A_{1}} \rightarrow B_{P_{L}}$ (see [3]). This reduces the existence of $A_{1}$-structure on a P. L. manifold to a bundle lifting problem.

Theorem 1. Any closed $A_{1}$-manifold is $P$. L. homeomorphic to a real algebraic variety.

Corollary 1. All P. L. manifolds of dimension less than 10 are P. L. homeomorphic to real algebraic varieties (also see [1]).

THEOREM 2. If a closed smooth manifold bounds a smooth spine-manifold, then it can be represented as a link of an isolated real algebraic singularity. (Converse of this is the Hironaka's resolution theorem.)

COROLlary 2. Elements of $\Gamma_{8}, 2 \Gamma_{10}$, and all exotic spheres which admit fixed point free smooth involutions are links of real algebraic singularities (also see [2]).

A BRIEF SKeTCH OF THE PROOFS. Let $M$ be a closed $A_{1}$-manifold. For simplicity assume $M^{m}=M_{0}^{m} \cup \operatorname{cone}\left(\Sigma^{m-1}\right)$; then there is $W^{m}$ with closed submanifolds $\left\{M_{i}\right\}$ such that $W / M_{i} \approx \operatorname{cone}(M)$, and $\partial W=\Sigma$. Let $\tilde{M}=M_{0} \cup W$.

By proving a relative version of the Nash-Tognoli approximation theorem we can make the smooth manifold $\widetilde{M}$ a real algebraic variety $V$, so that the smooth submanifolds $\left\{M_{i}\right\}$ of $\widetilde{M}$ correspond to the subvarieties $\left\{V_{i}\right\}$ of $V$.

AMS (MOS) subject classifications (1970). Primary 26A78; Secondary 57C25.

Key words and phrases. Algebraic varieties, exotic spheres.

1

Both authors supported by National Science Foundation grant MPS72-05055 A03. 
Let $V=f^{-1}(0)$ and $U V_{i}=g^{-1}(0)$, where $f(x)$ and $g(x)$ are polynomials. Let $F(x, t)=f(x)^{2}+(\operatorname{tg}(x)-1)^{2}$, then $\hat{F}(y)=|y|^{2 d} F\left(y /|y|^{2}\right)=0, y=$ $(x, t), d=\operatorname{degree} F$, gives the equations of

$$
V / V_{i} \approx \tilde{M} / M_{i}=M_{0} \cup\left(W / M_{i}\right) \approx M_{0} \cup \operatorname{cone}(\Sigma)=M .
$$

This sketches the idea of the proofs of Theorem 1 and 2. Corollary 1 and 2 are true because elements of $\Gamma_{8}, 2 \Gamma_{10}$ bound spine manifolds (see [4]); and any exotic sphere $\Sigma$ with fixed point free smooth involution $\tau$ bounds the obvious spine manifold $\Sigma \times I /(x, 0) \sim(\tau(x), 0)$.

\section{REFERENCES}

1. S. Akbulut, Real algebraic equations for a class of P. L. manifolds (to appear).

2. H. King, Approximating submanifolds of real projective space by varieties, Topology 15 (1976), $81-85$.

3. N. Levitt, Exotic singular structures on spheres, Trans. Amer. Math. Soc. 205 (1975), 371-388. MR 51 \# 14078.

4. R. Schult z, Circle actions on homotopy spheres bounding plumbing manifolds, Proc. Amer. Math. Soc. 36 (1972), 297-300. MR 46 \# 8248.

DEPARTMENT OF MATHEMATICS, UNIVERSITY OF WISCONSIN, MADISON, WISCONSIN 53706

DEPARTMENT OF MATHEMATICS, UNIVERSITY OF MARYLAND, COLLEGE PARK, MARYLAND 20742 